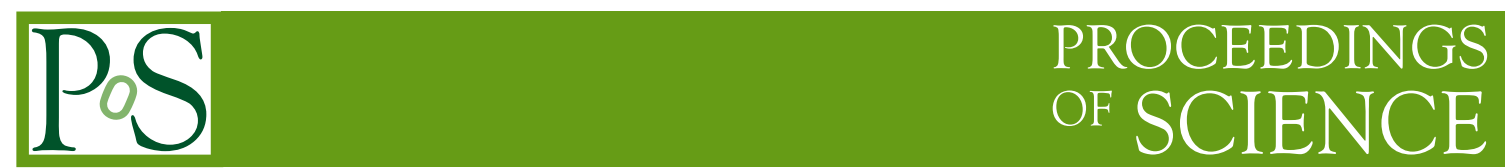

\title{
Nuclear Astrophysics in Underground Laboratories
}

\section{Marialuisa Aliotta*}

School of Physics and Astronomy, University of Edinburgh, Edinburgh, UK Scottish Universities Physics Alliance, SUPA, UK

E-mail: m.aliotta@ed.ac.uk

VI European Summer School on Experimental Nuclear Astrophysics, ENAS 6 September 18-27, 2011

Acireale Italy

* Speaker. 


\section{Introduction}

Stars generate energy and synthesise elements through nuclear reactions during quiescent evolution and fierce catastrophic explosions. Understanding the nucleosynthesis and the energyproduction mechanisms occurring at various astrophysical sites is at the heart of Nuclear Astrophysics. However, a detailed description of these processes is a truly multi-disciplinary effort which requires inputs from many diverse fields, including astronomical observations, theoretical stellar modelling, experimental nuclear physics, advanced detection techniques and instrumentation.

Despite impressive progress in the last few decades, many open questions still remain: What are the final stages of a massive star's lifetime? What determines whether the remnant of spectacular supernovae explosions is a neutron star or a black hole? How, when and where do stars synthesise the heavy elements which ultimately form much of our everyday environment? How can we explain the isotopic abundances observed in novae ejecta? The answers to these and similar questions invariably require accurate measurements of key nuclear processes at the energies at which they take place in stars. Unfortunately this is an extraordinarily difficult task.

Nuclear processes in stars are very rare events and reproducing them in the laboratory is a painstaking activity. In the best case scenarios, the cross sections of the relevant reactions are such that they produce sizeable effects in the detection systems (e.g. several tens of events per hour). More often, however, processes are as rare as one event per month or less. It then becomes of utmost importance to reduce to a minimum any source of unwanted background (typically induced by cosmic rays). Significant advances in this direction can then be achieved by the concurrent exploitation of cutting-edge instrumentation and measurements performed in deep underground laboratories.

In this lecture, I will present an outline of the experimental challenges encountered in studying nuclear reactions with low-energy stable beams and highlight the needs for underground facilities. I will also present an overview of the current status of key reactions in nuclear astrophysics and briefly describe some proposals for future underground laboratories.

\section{Why underground? Background sources and related issues}

Quiescent burning is largely dominated by reactions involving stable nuclei, on time scales of the order of billions of years. Thermonuclear reactions in stars take place over a narrow energy region, the so-called Gamow peak, well below the repulsive Coulomb barrier between the interacting nuclei. Hence, their cross-sections drop exponentially with decreasing energy and become increasingly difficult to measure in laboratories at the Earth's surface, where the signal-to-noise ratio is entirely dominated by natural and cosmic-ray induced background in the detection systems.

Although the nuclear cross section is a useful concept to determine the probability at which a given reaction takes place in the astrophysical plasma, what is measured in the laboratory is not the cross section, but rather the reaction yield, $\mathrm{Y}$, that is related to the cross section, $\sigma$, by:

$$
\mathrm{y}=\mathrm{N}_{\mathrm{p}} \mathrm{N}_{\mathrm{t}} \sigma \varepsilon
$$

where $\mathrm{N}_{\mathrm{p}}$ and $\mathrm{N}_{\mathrm{t}}$ are the number of projectile and target nuclei, respectively, and $\varepsilon$ the overall detection efficiency. For typical stable-beam experiments, one has $N_{p} \simeq 10^{14}$ pps (e.g. for a 100 
$\mu \mathrm{A}$ beam intensity and a $\mathrm{q}=1^{+}$charge state) and $\mathrm{N}_{\mathrm{t}} \simeq 10^{19}$ atoms $/ \mathrm{cm}^{2}$ (for solid state targets). Depending on whether the reaction under study produces charged particles or gamma rays, the overall detection efficiency $\varepsilon$ varies from as much as $\sim 100 \%$ to as little as $\sim 1 \%$, respectively. As for the cross sections involved, these are clearly given by nature and typically range between $10^{-18}$ barn $\leq \sigma \leq 10^{-9}$ barn at the extremely low energies of astrophysical relevance. Thus, for a characteristic cross sections of $10^{-15}$ barn, the measured reaction yields would typically be of the order of 0.3 to 30 counts/year! Such low yields are clearly extremely difficult to measure because concurrent background signals contribute to significantly reduce the signal-to-noise ratio. Maximising the experimental yield thus requires either improving the "signal", reducing the "noise", or a combination of both.

Improving the signal translates into the use of high beam currents and thicker and purer targets. There are, however, limitations as to the extent to which either can be achieved. Very high beam currents pose problems of charge confinement and heating effects on the targets (the latter can be cooled, but again there is a limitation as to how effectively heat can be dissipated away from the target). Increasing the target thickness will increase the reaction yield, but because of the exponential drop of the cross section, there is limited advantage in increasing the thickness beyond a certain limit. Also, high purity targets are extremely difficult and expensive to produce. For these reasons, most of the approaches aimed at improving the signal have already been exhausted on surface laboratories.

As far as background reduction is concerned, it is worth recalling that there are two main sources of background: beam induced and ambient. The former is produced by the beam through reactions with impurities in the target and/or reactions on beam line collimators and apertures. This source of background is difficult to minimise and the greatest care is devoted to the experimental setup design and to the production of the purest targets possible. The ambient background arises from: i) natural radioactivity (mainly from $U$ and and Th decay chains and from Rn contained in the laboratory building materials); ii) cosmic rays (muons, ${ }^{1,3} \mathrm{H},{ }^{7} \mathrm{Be},{ }^{14} \mathrm{C}, \ldots$ ); and iii) neutrons from $(\alpha, \mathrm{n})$ reactions and fission. Figure 1 shows a characteristic $\gamma$-ray background spectrum obtained at a surface laboratory. The strongest component to the observed $\gamma$ rays at $\mathrm{E}_{\gamma} \leq 3 \mathrm{MeV}$ is associated with the ambient natural radioactivity and displays intense characteristic lines. At higher energies the background remains rather flat and is mostly due to neutron-induced reactions and cosmic ray interactions with the experimental setup. This high-energy background can be reduced significantly by hosting the experiment in an underground environment. Depending on the depth of the location, the flux of cosmic muons can be reduced by several orders of magnitude compared to that received on the Earth's surface, thus almost suppressing the background at $\mathrm{E}_{\gamma} \geq 3.5 \mathrm{MeV}$. Further background reduction can be achieved by active or passive shielding of the detection system and in general the shielding is more effective underground than on the surface because of the already reduced cosmic-ray-induced interactions. Suppression of the background at lower energies is more difficult and the level of background will clearly depend on the geology of the underground site. An environment with a low $\mathrm{U}$ and $\mathrm{Th}$ content, such as, for example, that of a salt mine, would provide an ideal location. 


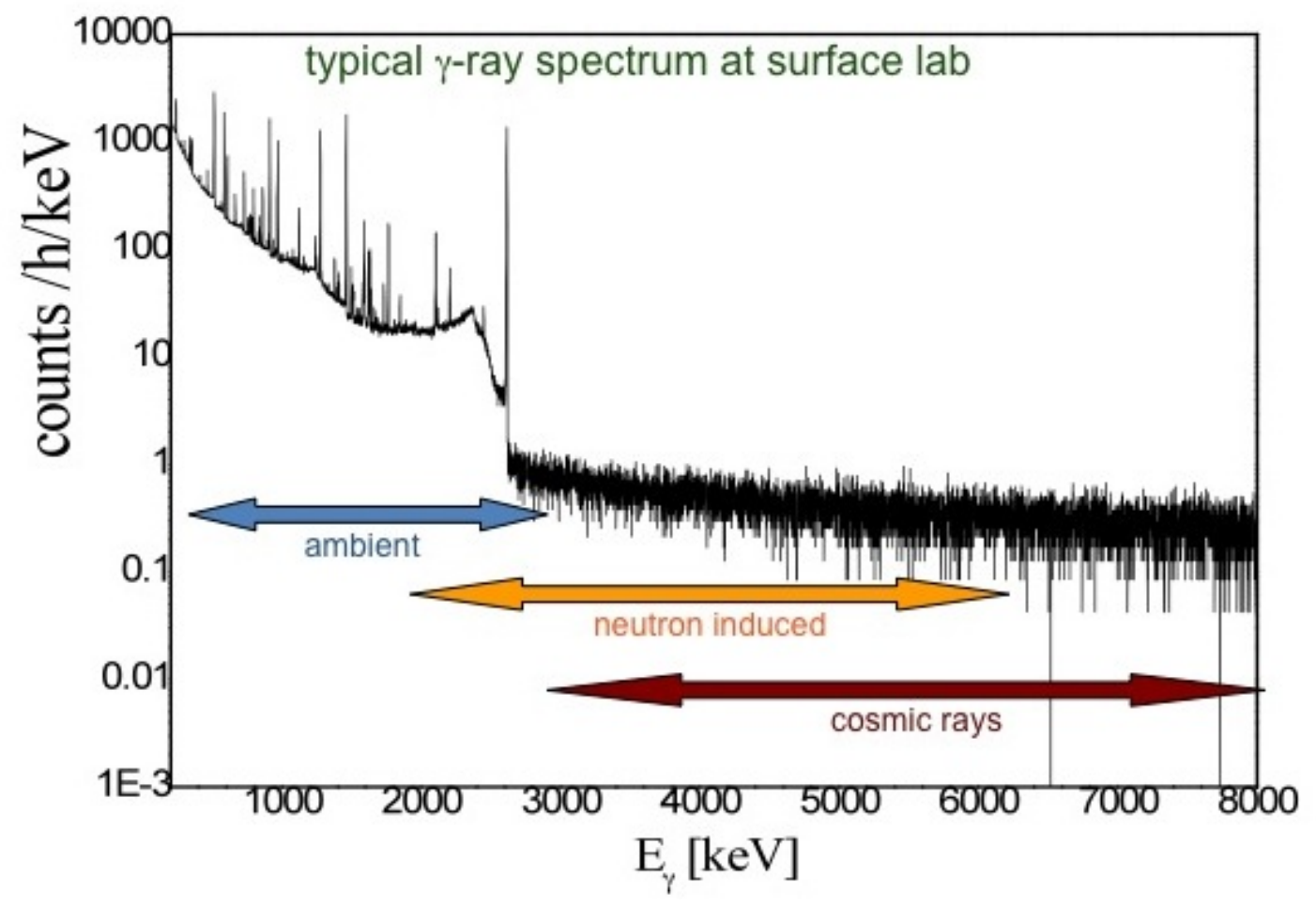

Figure 1: Typical gamma-ray background spectrum as measured on the Earth's surface. The background at $\mathrm{E}_{\gamma} \leq 3 \mathrm{MeV}$ arises from natural radioactivity, mainly associated with the decay of $\mathrm{U}$, Th and Rn contained in the laboratory building materials. At $\mathrm{E}_{\gamma} \geq 3 \mathrm{MeV}$ the background is mostly due to cosmic-rays interactions and neutron-induced reactions.

\section{LUNA: A pioneering project}

The potential of underground measurements has been dramatically demonstrated in the last two decades by the pioneering work performed at the LUNA (Laboratory Underground for Nuclear Astrophysics) facility located at the Laboratori Nazionali del Gran Sasso (LNGS) under the Gran Sasso massif (Italy). Here, the $1400 \mathrm{~m}$ rock overburden (or 3800 metre of water equivalent, m.w.e.) provides a cosmic-ray flux reduction factor of $10^{6}$ compared to a surface laboratory. Such a strong background reduction has enabled, for first time, the measurement of key reaction cross sections of astrophysical importance directly in their Gamow energy regions. For example, two fundamental reactions in the pp-chain, ${ }^{3} \mathrm{He}\left({ }^{3} \mathrm{He}, 2 \mathrm{p}\right){ }^{4} \mathrm{He}[1]$ and $\mathrm{d}(\mathrm{p}, \gamma){ }^{3} \mathrm{He}$ [2], were measured directly at solar Gamow-peak energies. Both measurements were carried out using a "homemade" $50 \mathrm{kV}$ accelerator capable of delivering protons and ${ }^{3} \mathrm{He}^{+}$beams of 300 to $500 \mu \mathrm{A}$ at energies between 10 and $50 \mathrm{keV}$. Following the early success of these measurements, the LUNA facility was upgraded by the acquisition of a commercial $400 \mathrm{kV}$ machine that became operational in 2002. Since then, LUNA has carried out investigations of other key reactions relevant to hydrogen burning in the pp-chain, the CNO cycles, the $\mathrm{MgAl}$ and NeNa cycles, and to the Big Bang nucleosynthesis (see $[3,4]$ for recent reviews). Perhaps the most impressive achievements of this extensive experi- 
mental programme are exemplified by the results on the ${ }^{14} \mathrm{~N}(\mathrm{p}, \gamma){ }^{15} \mathrm{O}$ reaction, the slowest of the CNO cycles reactions. The new improved data obtained at LUNA [5, 6] led to a reduction in the previously-accepted reaction rate by a factor 2 with a number of impressive consequences for many astrophysical scenarios. These include: a delay in the onset of the CNO cycle in massive stars; a reduction in the flux of CNO solar neutrinos (to the level of $2 \%$ instead of $4 \%$ as previously assumed) with important implications for the Borexino detector; and, most notably, an increase of about one billion years in the age of globular clusters (the oldest objects in our Galaxy) [7], and hence a corresponding increase in the age of the Universe, in better agreement with other determinations (see also [8] and references therein).

While the success of these measurements testifies to the enormous potential of underground environments, present capabilities at LUNA are limited both in ion species (mostly protons and helium isotopes) that can be produced and in the available energies. However, several open questions related to various stages of stellar evolution involve reactions with heavier nuclei (see below), which in turn would require a higher voltage machine. Thus, new upgraded facilities are clearly needed.

\section{Astrophysical reactions and open problems}

This section highlights some of the most important reactions relevant to open questions in stellar evolution and nucleosynthesis. In general, improvements on detection techniques at surface laboratories have already been exhausted and major advanced can only be expected to come from measurements at next-generation underground facilities.

\section{${ }^{12} \mathrm{C}(\alpha, \gamma){ }^{16} \mathrm{O}$}

This is perhaps the most important reaction in nuclear astrophysics as it plays a fundamental role in the evolution of any star during the helium-burning phase and determines the ratio between two fundamental elements: carbon and oxygen. At the typical Gamow peak energy for heliumburning, $\sim 300 \mathrm{keV}$, the expected cross section is of the order of $10^{-17}$ barn and is dominated by both ground-state transitions (through E1 and E2 contributions and interferences with $1^{-}$and $2^{+}$ states) and cascade transitions. In order to obtain a reliable extrapolation at low energies, the E1 and E2 amplitudes need to be fitted separately. Existing data sets extend to a minimum energy of about $1 \mathrm{MeV}$ and show systematic differences. At present, extrapolated S300 values differ by up to $100 \%$ and the calculated carbon abundance left by helium burning changes accordingly. Potentially, this is a good candidate for underground investigation, although advanced detection techniques would also be required.

\section{Carbon burning}

Carbon burning represents the third stage of stellar evolution for massive stars $\left(M \geq 8 \mathrm{M}_{\odot}\right)$ and proceeds mainly through the ${ }^{12} \mathrm{C}+{ }^{12} \mathrm{C}$ and, to a lesser extent, the ${ }^{12} \mathrm{C}+{ }^{16} \mathrm{O}$ fusion processes. In particular, the ${ }^{12} \mathrm{C}\left({ }^{12} \mathrm{C}, \mathrm{p}\right){ }^{23} \mathrm{Na}$ and the ${ }^{12} \mathrm{C}\left({ }^{12} \mathrm{C}, \alpha\right){ }^{20} \mathrm{Ne}$ channels critically govern the timescale of the carbon burning phase and influence later stages of stellar evolution. Hence, a knowledge of the reaction rates for these processes is essential to understand, for example, type Ia supernovae explosions and accreting neutron stars. Unfortunately, a reliable extrapolation into the Gamow peak is 
complicated by pronounced resonance features, observed down to the lowest energies measured so far $\left(\mathrm{E}_{\mathrm{cm}} \simeq 2.1 \mathrm{MeV}\right)$ ([9] and references therein). Direct measurements at even lower energies are therefore essential, but are severely hampered by both cosmic-ray and beam-induced backgrounds. Indeed, major improvements can be achieved by using advanced detection techniques (e.g. Compton suppressed HPGe detector array) ideally in underground measurements.

\section{Neutron sources}

Slow neutron-capture reactions (s-process) along the valley of beta stability are responsible for the origin of approximately $50 \%$ of all the elements beyond iron. One of the key open questions here regards the source of the stellar neutron flux available for the s-process. The most likely candidates are the reactions ${ }^{13} \mathrm{C}(\alpha, \mathrm{n}){ }^{16} \mathrm{O},{ }^{17} \mathrm{O}(\alpha, \mathrm{n}){ }^{20} \mathrm{Ne}$ and ${ }^{22} \mathrm{Ne}(\alpha, \mathrm{n}){ }^{25} \mathrm{Mg}$ and their low-energy cross-sections are crucial to determine the efficiency of the $s$-process under different stellar conditions. Only a few experiments have been performed to investigate these reactions and none of them was able to reach the relevant astrophysical energies. For the ${ }^{13} \mathrm{C}(\alpha, n){ }^{16} \mathrm{O}$ reaction, only energies down to $270 \mathrm{keV}$ were directly studied [10], whereas the energies of astrophysical relevance are around 170-200 keV. In order to distinguish between different S-factor extrapolations, a direct measurement of the ${ }^{13} \mathrm{C}(\alpha, \mathrm{n}){ }^{16} \mathrm{O}$ at $\mathrm{E}_{\alpha} \leq 270 \mathrm{keV}$ is highly desirable. For the ${ }^{22} \mathrm{Ne}(\alpha, \mathrm{n}){ }^{25} \mathrm{Mg}$ reaction, the measured reaction yield at $\mathrm{E}_{\alpha} \leq 800 \mathrm{keV}$ (i.e. the relevant astrophysical energies) is entirely due to cosmic-ray induced background [11]. As a consequence, the uncertainty in its rate increases to one order of magnitude below $\mathrm{T} \leq 0.3 \mathrm{GK}$, significantly affecting nucleosynthesis predictions. Similar conclusions also apply to the competing ${ }^{22} \mathrm{Ne}(\alpha, \gamma){ }^{26} \mathrm{Mg}$ reaction [12] and further low-energy measurements are clearly needed.

\section{Galactic nucleosynthesis}

Radiative capture reactions such as ${ }^{22} \mathrm{Ne}(\mathrm{p}, \gamma){ }^{23} \mathrm{Na},{ }^{23} \mathrm{Na}(\mathrm{p}, \gamma){ }^{24} \mathrm{Mg}$ and ${ }^{26} \mathrm{Al}(\mathrm{p}, \gamma){ }^{27} \mathrm{Si}$ significantly influence the production of $\mathrm{Ne}, \mathrm{Na}, \mathrm{Mg}$ and $\mathrm{Al}$ in Asymptotic Giant Branch stars [13]; while processes such as ${ }^{17,18} \mathrm{O}(\mathrm{p}, \gamma){ }^{18,19} \mathrm{~F},{ }^{33} \mathrm{~S}(\mathrm{p}, \gamma){ }^{34} \mathrm{Al}$ and many other proton-induced reactions on $\mathrm{A}=20-40$ nuclei are crucial to the production of key isotopes often observed in novae ejecta (see $[14,15]$ for a review). Once again, essential improvements in the measurement of all the above reactions could be achieved by the use of advanced detection systems, ideally in underground laboratories.

\section{Proposals for underground laboratories}

A large number of underground laboratories exists worldwide for the investigation of rare physical phenomena, from neutrino-less $\beta \beta$ decay, to neutrino's properties and detection, dark matter search, proton radioactivity, and gravitational waves (see [16] for an overview of existing underground facilities and a description of their physical characteristics). Unfortunately, only few of these would meet the requirements for easy access, dedicated space, and suitable geology, required for nuclear astrophysics experiments. Thus, LUNA remains at present the only underground accelerator in the world specifically dedicated to Nuclear Astrophysics. Given the importance of further underground measurements, many projects are currently being proposed for future underground facilities both in Europe and elsewhere. In Europe, the need for further facilities has been recognised 
and fully endorsed by the international community, as summarised in the recent NuPECC Long Range Plan 2010: The effort to put into operation a machine of several MV in a European deep underground laboratory should be considered with the highest priority. This could be achieved in the next three to five years with the opportunity to measure one or two key reactions within the next decade. Considering the high scientific interest in measuring several more nuclear reactions, the case could be made to complete the programme with a second facility designed for a complementary set of measurements. [17].

Here, I will present a brief overview of some relevant proposals in Europe and in the United States. However, the map of underground science is rapidly evolving and future facilities may be proposed in the next few years.

\section{Europe}

\section{LUNA MV}

This project foresees the upgrade of the existing LUNA facility by the acquisition of a MV machine capable of accelerating light ion species (protons, deuteron, and helium isotopes). A Letter of Intent (LoI) for a long term scientific programme based on a new accelerator has been presented to the Laboratori Nazionali del Gran Sasso. The proposed measurements cover a broad range of reactions occurring in helium-burning stars. The development of a full project for the preparation of the site inside LNGS (floor sealing, ventilation, power supply, ...) is almost complete and the shielding of the experimental hall from the rest of the laboratory is under study. Simulations on the expected neutron fluxes have been carried out and technical solutions are currently being considered. Further details on the current status of the LUNA MV upgrade can be found in the contribution of A. Guglielmetti at this Summer School ${ }^{1}$.

\section{Canfranc}

The Canfranc Underground Laboratory (LSC) is a new facility for Underground Science, conceived as a Consortium of the Spanish Ministry of Science and Innovation, the Aragon Regional Government and the University of Zaragoza. The laboratory is located under the Pyrenees mountain "El Tobazo", where the rock over burden provides 2500 meters-water-equivalent shielding from cosmic rays and offers a low background environment for the next generation of experiments in particle and astro-particle physics [18].

The nuclear astrophysics project foresees the installation of a 3.5MV accelerator coupled with a RF source for the production of protons and alpha particles beams. However, the possibility of a future upgrade to an ECR source for the acceleration of heavier nuclei (e.g. ${ }^{12} \mathrm{C}$ and ${ }^{16} \mathrm{O}$ ) is also being considered. This solution, however, may involve considerable R\&D development to meet the stringent requirements of nuclear astrophysics experiments (high currents, high long-term stability, low energy dispersion, etc). For the installation of the machine and its related instrumentation the site requires the construction of an independent and well isolated cave. At the time of writing, the pre-engineering design for the new cave has been completed and permit for excavation is expected during 2012, after which excavation work may start. Neutron measurements and simulations have

\footnotetext{
${ }^{1}$ At the time of writing, a proposal in support of the LUNA MV programme has just been submitted to the EU under the Synergy Grants scheme, and a "Progetto Premiale" has also been submitted to the Italian Research Ministry.
} 
recently been completed and the results will soon be submitted for publication [19]. Funds for the accelerator are being sought in parallel. A full Letter of Intent will be presented to the LSC Scientific Committee in the next few months.

\section{Boulby}

For completeness of information, it is also worth mentioning the opportunity offered by the salt and potash mine at Boulby (UK) for hosting an accelerator facility specifically designed and dedicated to experimental Nuclear Astrophysics. The depth of the Boulby mine (1.1 km below surface) guarantees a six-order-of-magnitude reduction in the muon flux. Also, the mine's extension and the composition of its salt environment, provide a unique site with ideal features for nuclear astrophysical studies. These include:

- a much lower background (typically by a factor of 5-50 in the $\mathrm{E}_{\gamma} \leq 3 \mathrm{MeV}$ ) than in other types of sedimentary rocks (particularly those high in U/Th content);

- no space constraints, thanks to the availability of large areas for the construction of the infrastructure (approximately 150-200 $\mathrm{m} 2$ needed) and, hence, no problems of interference with other scientific programs (at present Dark Matter only);

- no constraints on the beam species, and/or reaction products, which can be generated;

- safety and support infrastructure already in place and well established.

Indeed, all these features would make Boulby an ideal location for the installation of a worldleading accelerator facility, and a proposal for an underground facility at Boulby (UK) was submitted to the UK STFC research council in 2009 [20]. Unfortunately, following the severe financial cuts to science in the UK over the last few years, the proposal did not get funding despite receiving extremely positive refereeing.

\section{Felsenkeller}

The Felsenkeller area is located in an ancient quarry in the Weißeritz valley, $5 \mathrm{~km}$ from Dresden, Germany. The underground facility consists of nine tunnels dug in the 1850s to host the ice cellar of the nearby Felsenkeller brewery. The average rock overburden is $47 \mathrm{~m}$, equivalent to $110 \mathrm{~m}$ water (m.w.e.), leading to a reduction of the muon flux by a factor of 30-50 compared to surface's. As part of a staged approach, the use of a shallow-underground site at Felsenkeller, Dresden (Germany) is currently being considered. The project would encompass bringing a used accelerator to this already-existing facility, greatly speeding up progress and reducing cost. However, the background at Felsenkeller is a factor of 3-10 higher than at Gran Sasso, meaning that the most challenging measurements would still have to be done deep underground. The results of comparative measurements between the background levels obtained at the Earth's surface, deep underground at LUNA, and at the Felsenkeller shallow-underground facility have recently been published [21].

All these activities in Europe clearly demonstrate the widespread interest within the nuclear astrophysics community for new underground facilities. To further raise awareness within the community and also in an effort to coordinate various initiatives, a series of International Workshops on 
Underground Laboratories have taken place over the last few years. The first of these workshops was held in Barcelona in 2009. Follow-up meetings took place in Dresden in 2010, and at LNGS, Gran Sasso in 2011, while the next workshop will be in Canfranc on 22-23 March 2012.

\section{United States}

\section{DIANA}

In the United States, plans to construct an underground facility at the site of the former gold mine of Homestake in South Dakota are under way. Specifically, the DIANA project (Dakota Ion Accelerators for Nuclear Astrophysics) is a collaboration between the University of Notre Dame, University of North Carolina, Western Michigan University, and Lawrence Berkeley National Laboratory to build a nuclear astrophysics accelerator facility $1.4 \mathrm{~km}$ below ground as part of the Deep Underground Science and Engineering Laboratory (DUSEL) proposal. DIANA would consist of two high-current accelerators: a 30 to $400 \mathrm{kV}$ variable, high-voltage platform; and a second, dynamitron accelerator with a voltage range of $350 \mathrm{kV}$ to $3 \mathrm{MV}$. The project foresees the development of a high-density super-sonic jet-gas target capable of delivering up to $100 \mathrm{~mA}$ on the low energy accelerator and several $\mathrm{mA}$ on the high energy accelerator. As a unique feature, both accelerators are planned to be equipped with either high-current microwave ion sources or multi-charged ECR ion sources producing ions from protons to oxygen. The design work is underway for both the accelerator and jet-gas target [22], but the funding situation is somewhat unclear at present [23].

\section{Rest of the world}

In addition to the projects and proposals already mentioned, a number of other underground laboratories are currently being planned or built at several other locations worldwide. Here, we just mention two that may potentially be suitable to host nuclear astrophysical studies. One such project exploits the construction of a low altitude tunnel as part of a strategic improvement of the Agua Negra pass in the Andes, near the Argentina-Chile border. The ANDES underground laboratory would be the only such laboratory in the southern hemisphere. The laboratory would consist of 3 halls shielded by $1750 \mathrm{~m}$ of rock from cosmic rays. A linear access tunnel would be available for the installation of a linear particle accelerator for nuclear astrophysics studies [24, 23]. If the proposed project goes ahead, the opening of the laboratory would be concurrent with the opening of the Agua Negra tunnel in 2018 [24].

Finally, it is worth mentioning the recent opening of China's first extremely deep underground laboratory, the China Jinping Underground Laboratory (CJPL), which started operation on December 12th, 2010. Jointly constructed by Tsinghua University and Ertan Hydropower Development Company (Ertan), the laboratory was excavated from a 17-kilometer transporting tunnel in Jinping Mountain. Covered by 2,400 meters of rock, CJPL is now the deepest underground laboratory in the world. At present, research activities are mostly focussed on dark matter search [25].

\section{Summary and outlook}

Thermonuclear reactions provide the engine that makes stars shine and the furnace that synthesise the elements beyond primordial hydrogen and helium. The direct investigation of these reactions in terrestrial laboratories is severely hampered by the large background induced by cosmic 
rays' interaction with the experimental detection systems. Often, the only solution to significantly improve the signal-to-noise ratio consists in performing cross-section measurements in deep underground laboratories where the cosmic-ray flux can be reduced by several orders of magnitude with respect to laboratories at the Earth's surface. The potential for underground measurements has been demonstrated by the pioneering work performed at LUNA, in Gran Sasso (Italy). Yet, despite the major advanced obtained so far, many important astrophysical reactions remain beyond current capabilities at LUNA. In this lecture, I have outlined some of the key open questions in nuclear astrophysics and have presented an overview of current plans for future next-generation underground facilities both in Europe and elsewhere. It is hoped that some of the proposed facilities will be realised in the near future so as to open up new and unprecedented opportunities for major breakthrough in this fascinating research field.

\section{References}

[1] R. Bonetti et al., Phys. Rev. Lett. 82 (1999) 5205

[2] C. Casella et al., Nucl. Phys. A 706 (2002) 203

[3] C. Broggini et al., Annu. Rev. Nucl. Part. Sci. 60 (2010) $53 Đ 73$

[4] H. Costantini et al., Rep. Prog. Phys. 72 (2009) 086301

[5] A. Formicola et al., Phys. Lett. B59 (2004) 61

[6] A. Lemut et al., Phys. Lett. B 634 (2006) 483

[7] G. Imbriani et al., Astron. \& Astrophys. 420 (2004) 625

[8] F. Strieder \& C. Rolfs, Prog. in Part. and Nucl. Phys. 59 (2007) 562-578

[9] T. Spillane et al., Phys. Rev. Lett. 98 (2007)122501

[10] M. Heil et al., Phys. Rev. C 78 (2008) 025803

[11] M. Jaeger et al., Phys. Rev. Lett. 87 (2001) 202501

[12] A. Karakas et al., Astrophys. Journ. 643 (2006) 471

[13] R.G. Izzard et al., Astron. \& Astrophys. 466 (2007) 641-648

[14] C. Iliadis et al., Astrophys. Journ. S134 (2001) 151

[15] C. Iliadis et al., Astrophys. Journ. S142 (2002) 105

[16] E. Coccia, Journ. Phys. Conference Series 203 (2010) 012023

[17] http://www.nupecc.org/lrp2010/Documents/lrp2010_final_hires.pdf

[18] (http://www.lsc-canfranc.es/)

[19] L.M. Fraile, Private Communication (2012)

[20] M. Aliotta, for the ELENA Collaboration: Proposal for a feasibility study and design (2009)

[21] T. Szücs et al., Eur. Phys. J. A48 (2012) 8

[22] D. Leitner et al. Proceedings of 2011 Particle Accelerator Conference, New York, NY USA, and http://www.jinaweb.org/dusel/DIANA/rpt76801.pdf (January 26, 2012) 
[23] M. Wiescher, Private Communication (2012)

[24] http://fisica.cab.cnea.gov.ar/particulas/andes/main.php (January 28, 2012)

[25] Q. Yue and H.T. Wong, http://arxiv.org/pdf/1201.5373.pdf (January 28, 2012) 